\title{
Factores de Riesgo Asociados a Parásitos Gastrointestinales en Animales de Producción
}

\section{Risk Factors Associated with Gastrointestinal Parasites in Production Animals}

\author{
E. G. Quiroga-Calderón', A. B. Gatica-Colima1, Z. Carlo-Rojas ${ }^{1}$
}

1 Universidad Autónoma de Ciudad Juárez

\section{RESUMEN}

El análisis de los factores de riesgo que afectan la salud de los animales incluye la identificación de organismos parásitos y su posible efecto en la salud y entorno del animal. El objetivo de este estudio es identificar y evaluar el impacto de los factores de riesgo que están asociados a la prevalencia de parásitos gastrointestinales en animales de producción del Rancho Universitario de la Universidad Autónoma de Ciudad Juárez durante la temporada de verano de 2020, por medio de la Matriz de Leopold combinada con los Criterios Relevantes Integrados (CRI). Las prevalencias por géneros de helmintos fueron: Trichostrongylus (66\%) y Strongyloides (16\%) en bovinos, Haemonchus (40\%) en ovinos, Strongyloides (66\%) en porcinos, Strongylus (100\%) y Cyathostomum (66\%) en equinos y Ascaris (100\%) en gallinas. La implementación propuesta de estas metodologías adaptadas a las condiciones complejas y particulares permitió identificar y categorizar el impacto de los factores de riesgo en la salud de los animales de producción. Ausencia de pediluvio, falta de desparasitación y animales nuevos sin cuarentena resultaron factores de riesgo de tipo severo, los cuales afectaron a todos los grupos de animales del sitio de estudio, por lo que requieren atención prioritaria.

PALABRAS CLAVE: helminto; análisis de riesgo; impacto; salud.

\section{ABSTRACT}

The analysis of risk factors affecting the health of animals includes the identification of parasitic organisms and their possible effect on the health and environment of the animal. This study was aimed to identify and evaluate the impact of risk factors associated with the prevalence of gastrointestinal parasites in production animals of the University Ranch of the Universidad Autónoma de Ciudad Juárez during the 2020 summer season using the Leopold's Matrix combined with the Integrated Relevant Criteria. The prevalences by genus of helminths were: Trichostrongylus (66\%) and Strongyloides (16\%) in cattle, Haemonchus (40\%) in sheep, Strongyloides (66\%) in pigs, Strongylus (100\%) and Cyathostomum (66\%) in horses and Ascaris (100\%) in chickens. The proposed implementation of these methodologies adapted to complex and particular conditions made it possible to identify and categorize the impact of risk factors on the health of production animals. Absence of footbath, lack of deworming and new animals without quarantine were risk factors of a severe type, which affected all groups of animals in the study site, therefore requiring priority attention.

KEYWORDS: helminth; risk analysis; impact; health.

Correspondencia:

DESTINATARIO: Zarhelia Carlo Rojas

INSTITUCIÓN: Universidad Autónoma de Ciudad Juárez / Instituto de Ciencias Biomédicas

DIRECCIÓN: Anillo Envolvente del Pronaf y Estocolmo s/n, col.

Progresista, C. P. 32310, Ciudad Juárez, Chih., México

CORREO ELECTRÓNICO: zarhelia.carlo@uacj.mx
Fecha de recepción: 14 de septiembre de 2021. Fecha de aceptación: 25 de septiembre de 2021. Fecha de publicación: 1 de octubre de 2021.
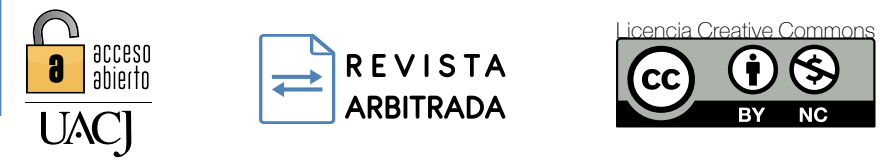

*El presente artículo se deriva de la investigación "Factores de riesgo asociados a la prevalencia de parásitos gastrointestinales en animales de producción del Rancho Universitario de la UACJ", de Elkin Giovanny Quiroga Calderón, la cual fue galardonada por la Coordinación General de Investigación y Posgrado de la Universidad Autónoma de Ciudad Juárez por ser el mejor trabajo del Coloquio Institucional de Posgrado 2020, en la mesa Procesos Productivos. 


\section{INTRODUCCIÓN}

El análisis de los factores de riesgo que afectan la salud de los animales permite la identificación de un agente infeccioso y su posible impacto en la salud y el entorno [1], así como la adopción de medidas de control para reducir el riesgo y aumentar la bioseguridad [2]. Un factor de riesgo es considerado como la posibilidad de padecer un suceso de tipo perjudicial que afecta la salud de un individuo ${ }^{[3]}$.

Un agente infeccioso puede ser un parásito gastrointestinal que puede causar síntomas de malestar, pérdida de peso, diarrea, anemia y edema [4]. Algunos de estos parásitos suelen ser zoonóticos, lo cual representa una problemática de salud pública ${ }^{[5]}$.

Dentro de los parásitos gastrointestinales que afectan a las distintas especies animales se encuentran los helmintos, que infectan a una gran cantidad de especies domésticas y silvestres que, a su vez, funcionan como hospederos [6]. Otros parásitos son los protozoos, de ambientes acuosos y suelo [7], cuyas infecciones ocasionan problemas de salud animal y pérdidas económicas [8].

Obanda et al. ${ }^{[9]}$ mencionan que la convivencia e interacción dentro de un mismo hábitat entre diversas especies animales e incluso la humana, pueden contribuir al riesgo de transmisión parasitaria gastrointestinal. Duffy et al. [10] señalan que cuando diversas especies hospederas llegan a compartir recursos dentro de un mismo hábitat, especialmente agua y pasto, esta interacción puede influir en las tasas de contacto y transmisión parasitaria debido a que las etapas infecciosas de los parásitos a menudo se dispersan fecalmente en el ambiente y dependen de las condiciones climáticas y ambientales para su propagación, persistencia y transmisión.

En el Valle de Juárez, Chihuahua, México, se encuentra el Rancho Universitario de la Universidad Autónoma de Ciudad (RU-UACJ), en el que coexisten diferentes especies en sistemas de producción animal. Ahí se puso a prueba una metodología para evaluar el impacto de los factores de riesgo asociados a la prevalencia de parásitos gastrointestinales en los animales de producción durante el verano de 2020, lo cual ayudará a establecer un modelo de control que disminuya el riesgo potencial detectado. Este trabajo presenta un avance de un estudio de mayor lapso.

\section{METODOLOGÍA}

\section{A. Ubicación del área de estudio}

El desarrollo del estudio se llevó a cabo en el RU-UACJ, situado en el Valle de Juárez, municipio de Praxedis G. Guerrero, Chihuahua, en el km 64 de la vía Juárez-Porvenir. La zona presenta una altitud de $1090 \mathrm{msnm}$ y coordenadas geográficas de referencia $31^{\circ} 21^{\prime} 20^{\prime \prime} \mathrm{N} \mathrm{y}$ $105^{\circ} 59^{\prime} 59^{\prime \prime} \mathrm{O}$ [11]

El RU-UACJ se caracteriza por ser un recinto multidisciplinar en áreas agropecuarias y cuenta con animales de producción como bovinos, ovinos, caprinos, porcinos, equinos y aves de corral [12].

\section{B. Identificación de parásitos gastrointestinales en muestras de excremento}

Durante tres días consecutivos del verano de 2020 se tomaron las muestras de excremento de bovinos, ovinos, caprinos, porcinos, equinos y asno recolectadas directamente del recto de los animales, utilizando guantes para minimizar cualquier tipo de contaminación. En las gallinas se recolectó del piso una muestra compuesta de excremento después de la defecación espontánea.

Las muestras se depositaron en bolsas de polietileno rotuladas y refrigeradas en hielera a una temperatura de 4 ${ }^{\circ} \mathrm{C}[13]$. Posteriormente, se transportaron al Laboratorio de Ciencias Básicas de la División Multidisciplinaria de la UACJ en Nuevo Casas Grandes, donde se les aplicó las técnicas coproparasitoscópicas de Faust, sedimentación y McMaster [14]. En la Tabla 1 se detalla el número de muestras de excremento recolectado de los animales de producción, lo cual se determinó por medio de la fórmula para población finita ${ }^{[15]}$.

TABLA 1

Número de Muestras de Excremento

\begin{tabular}{|l|c|}
\hline \multicolumn{1}{|c|}{ ANIMALES DE PRODUCCIÓN } & NÚMERO DE MUESTRAS \\
\hline Bovinos & 12 \\
\hline Ovinos & 15 \\
\hline Caprinos & 13 \\
\hline Porcinos & 6 \\
\hline Equinos & 3 \\
\hline Asnales & 1 \\
\hline Gallinas & 1 muestra compuesta \\
\hline
\end{tabular}


Mediante la Ecuación (1) se determinó el porcentaje de prevalencia para cada grupo animal ${ }^{[16]}$.

$$
P=\left(\frac{n}{N}\right) \cdot 100
$$

donde $P=$ prevalencia, $n=$ número de animales positivos, $N=$ total de animales.

\section{Identificación y determinación de los factores de riesgo}

La identificación y determinación de los factores de riesgo se realizó mediante una evaluación de bienestar animal, basada en los principios de libertad de Welfare Quality [17]. Para ello, solo se tuvo en cuenta siete criterios que pueden estar asociados a una infección parasitaria gastrointestinal, condición corporal, limpieza de bebederos, estiércol en el cuerpo, limpieza y comodidad alrededor de la zona de descanso, cojeras, heridas en el cuerpo e infecciones locales, asignando un valor de 1 , 3 y 5 . De acuerdo a la escala valorativa, "1" corresponde a una situación negativa, es decir, no puede haber más deficiencias en el bienestar; " 3 " corresponde a una situación neutral y " 5 " a una situación positiva. Cabe resaltar que para el criterio de condición corporal se valoró lo siguiente: animales con condición corporal normal " 3 ", animales con condición corporal delgada " 1 " y animales con condición corporal con sobrepeso " 5 ".

Asimismo, se utilizó una lista de revisión [18], a partir de la literatura, en cuanto a prácticas que pueden generar un cuadro de infección parasitario gastrointestinal, entre ellas ausencia de tapete sanitario, no desparasitación, animales nuevos sin previa cuarentena, manejo inadecuado de animales muertos, manejo inadecuado de animales heridos, accesibilidad con fauna silvestre y pastoreo mixto. Estas se cotejaron en el RU-UACJ para comprobar o descartar su aplicabilidad.

Todos los criterios y prácticas identificados como inadecuados se determinaron como factores de riesgo y se incluyeron en una matriz para evaluar su impacto y conocer su grado de severidad sobre los animales de producción del RU-UACJ.

\section{Evaluación de impacto}

Se evaluaron los impactos de los factores de riesgo aplicando el método de Matriz de Leopold combinado con los Criterios Relevantes Integrados formulado por Buroz en 1994 (citado por [19]), para lo cual se asignaron valores a los factores de riesgo de acuerdo a los criterios de Intensidad $(I)$, Extensión $(E)$, Duración $(D)$, Reversibilidad $(R)$ e Incidencia $(G)$, en una escala de valores comprendida entre 1 y 10 para todos los criterios, con más impacto para valores altos [19].

Para la asignación de los valores de cada factor, se procedió a calcular su Magnitud ( $M$ ) [Ecuación (2)] y el Valor del Índice Ambiental (VIA) o importancia de cada factor [Ecuación (3)]. Cabe resaltar que cada criterio tiene asignado un valor de peso, establecido de acuerdo a la metodología desarrollada en Gayoso [19] (Tabla 2).

$$
\begin{gathered}
M=(I \cdot W I)+(E \cdot W E)+(D \cdot W D) \\
V I A=\left(R^{W R} \cdot G^{W G} \cdot M^{W M}\right)
\end{gathered}
$$

TABLA 2

Valores de Peso para cada Criterio, Método de Criterios RELEVANTES INTEGRADOS [19]

\begin{tabular}{|l|c|c|}
\hline \multicolumn{1}{|c|}{ Criterio } & ClaVe & VALOR DE PESO \\
\hline Intensidad & $W I$ & 0.40 \\
\hline Extensión & $W E$ & 0.40 \\
\hline Duración & $W D$ & 0.20 \\
\hline Reversibilidad & $W R$ & 0.22 \\
\hline Incidencia & $W G$ & 0.17 \\
\hline Magnitud & $W M$ & 0.61 \\
\hline
\end{tabular}

Por último, se procedió a calcular la severidad $(S)$ de cada factor mediante la Ecuación (4).

$$
S=M \cdot V I A
$$

La $S$ de impacto de un factor de riesgo se calificó de acuerdo a la siguiente escala:

$\begin{array}{ll}\text { Leve } & 1-5 \\ \text { Moderado } & 6-15 \\ \text { Severo } & 16-39 \\ \text { Crítico } & 40-100\end{array}$

\section{RESULTADOS Y DISCUSIÓN}

\section{A. Identificación de parásitos gastrointestinales en los animales de producción del RU-UACJ}

Se analizaron 51 muestras de excremento, identificando seis géneros de parásitos gastrointestinales del filo Nematoda, de las cuales 22 fueron positivas a uno o dos géneros, representando el $43.1 \%$, mientras que 29 
muestras fueron negativas $56.9 \%$. Cuatro muestras fueron positivas a dos géneros, representando el 7.9\%, y 18 muestras fueron positivas a un solo parásito, representando el 35.2\%. En la Tabla 3 se describe la identificación de los géneros de parásitos gastrointestinales hallados durante la estación de verano de 2020 y sus respectivos porcentajes de prevalencia parasitaria.

TABLA 3

Identificación y Prevalencia de Huevos de Parásitos (VERANO 2020)

\begin{tabular}{|l|c|c|c|}
\hline $\begin{array}{c}\text { AnIMALES DE } \\
\text { Producción }\end{array}$ & $\begin{array}{c}\text { GÉnERo DE } \\
\text { PARÁsito }\end{array}$ & $\begin{array}{c}\text { PARASITAdo / } \\
\text { No PARASITADo }\end{array}$ & $\begin{array}{c}\text { PREVALENCIA } \\
(\%)\end{array}$ \\
\hline Gallinas & Ascaris & + & 100 \\
\hline Equinos & Strongylus & $3 / 0$ & 100 \\
& Cyathostomum & $2 / 1$ & 66 \\
\hline Porcinos & Strongyloides & $4 / 2$ & 66 \\
\hline Bovinos & Trichostrongylus & $8 / 4$ & 66 \\
& Strongyloides & $2 / 10$ & 16 \\
\hline Ovinos & Haemonchus & $6 / 9$ & 40 \\
\hline Caprinos & - & $0 / 13$ & - \\
\hline Asno & - & $0 / 1$ & - \\
\hline
\end{tabular}

Símbolos: -, no se reportaron parásitos; +, solo se reportó como positivo.

Los géneros de parásitos que predominaron con prevalencias de 100\% fueron Strongylus en équidos y Ascaris en gallinas.

Durante la revisión de literatura en la región se encontró información de parásitos gastrointestinales en coyotes ${ }^{[20]}$, agua ${ }^{[21]}$, ganado lechero ${ }^{[22]}$, comunidades de personas ${ }^{[23]}$ y grandes felinos en cautiverio [24]. Estos registros permiten establecer la presencia en la zona de los diversos helmintos, en una amplia de hospederos y en el ambiente.

La detección de Strongyloides en porcinos y bovinos fue de $66 \%$ y $16 \%$ en el presente estudio, en coincidencia con lo reportado por Petters [20], en coyotes en el Área Natural Protegida Médanos de Samalayuca, Chihuahua, México, en la temporada cálida de 2019. Aunque son diferentes especies y cargas parasitarias, el hallazgo de este helminto en ambos estudios evidencia que es común en la zona e infecta a una diversidad de vertebrados. Por tal razón, la interacción entre animales de producción y fauna silvestre hace factible una transmisión parasitaria. La presencia de este parásito en los bovinos y porcinos del RU-UACJ probablemente se relacione con los coyotes que normalmente frecuentan los alrededores y algunas áreas del RU-UACJ, lo que puede favorecer a una transmisión bidireccional entre los animales de producción y silvestres [6]. Otra razón puede ser que la forma de transmisión es percutánea; los animales de producción en el RU-UACJ permanecen la mayoría del tiempo confinados y la limpieza de los corrales es inconstante, lo cual favorece a un mayor riesgo de infección en los animales.

En este estudio, la presencia a huevos de Ascaris resultó positiva en la muestra compuesta de gallinas. De acuerdo con los resultados de Maya [21], fue el género de huevos de helminto más frecuente en agua tratada, residual y de uso agrícola en Nuevo Casas Grandes, Chihuahua, región cercana que comparte similar clima. Otros géneros reportados en agua en ese trabajo fueron Toxocara, Hymenolepis, Dipylidium, Fasciola, Taenia y Necator, aunque por la alta frecuencia presentada, Ascaris se ubicó entre los riesgos a la salud en relación al contacto con agua tratada o residual empleada en sistemas de producción agropecuarios. Resulta recomendable un monitoreo de las aguas empleadas en la irrigación para caracterizar, en su caso, la infestación por calidad del agua y minimizar riesgos.

En contraste con los géneros parasitarios obtenidos en el presente estudio con enfoque a helmintos, Quezada et al. [22] trabajaron con métodos dirigidos a protozoarios en Ciudad Juárez, Chihuahua, México, y reportaron ooquistes de Coccidias en becerras post destete, con una prevalencia del 62\% durante el otoño de 2013 . Generalmente, este parásito afecta al ganado bovino en sistemas intensivos, siendo los semovientes jóvenes los más susceptibles debido a factores extrínsecos e intrínsecos del animal [25].

Por otro lado, Flores et al. [23], en el Valle de Juárez, Chihuahua, México, realizaron un estudio epidemiológico en personas, en el que reportaron prevalencias de Giardia lamblia, con un $67.18 \%$, seguido por Cryptosporidium parvum, 34.37\%, y Cyclospora cayetanensis, $21.87 \%$, en heces humanas debido a deficiencias en las condiciones sanitarias de los pobladores de esta zona. Las explotaciones pecuarias locales también tienen deficiencias en sus condiciones sanitarias y en las acciones de manejo acorde al nivel sociocultural de la zona, lo que sugiere una perspectiva de altos niveles de parasitosis.

González [24] documentó huevos de Toxocara spp. y Toxascaris leonina en muestras de excremento de león 
(Panthera leo), puma (Puma concolor) y jaguar (Panthera onca) en el Zoológico San Jorge, ubicado al este de Ciudad Juárez. De acuerdo con Aranda et al. [26], la presencia de estos helmintos en los felinos puede corresponder a la ingesta de larvas infectantes presentes en el ambiente. Por ello, una evaluación integral, incluyendo agua y suelo, es pertinente para comprobar la presencia de Toxocara spp. en el suelo y agua de riego, además de descartar su presencia de canes y fauna silvestre en áreas de interacción con ganado.

Los parásitos identificados en el presente estudio, junto con los de Petters [20], Maya [21], Quezada et al. [22], Flores et al. [23] y González [24], indican que los parásitos están circulando a lo largo del Valle de Juárez y sus zonas cercanas, lo cual se puede considerar como un potencial de riesgo de infección para los animales que habitan en el RU-UACJ y sus alrededores.

\section{B. Identificación y determinación de los factores de riesgo}

En la Tabla 4 se observa la calificación de cada criterio de la evaluación de bienestar animal. Durante la limpieza de bebederos se encontraron gallinas dentro del recipiente del bebedero, mientras que en los rumiantes y équidos se hallaron bebederos adecuados pero sucios. Estas razones pueden favorecer el contagio de parásitos gastrointestinales, ya que se detectó Ascaris en gallinas, Trichostrongylus y Strongyloides en bovinos, Haemonchus en ovinos y Cyathostomum y Strongylus en equinos.

TABLA 4

Calificación de los Criterios Identificados y Determinados como FaCtores de Riesgo mediante la Evaluación de Bienestar Animal

\begin{tabular}{|c|c|c|c|c|c|c|c|}
\hline \multirow{2}{*}{ Criterio } & \multicolumn{7}{|c|}{ ANIMALES DE PRODUCCIÓN } \\
\hline & GALLINAS & EQUINOS & Porcinos & Bovinos & Ovinos & CAPRINOS & Asno \\
\hline Limpieza de bebederos & 1 & 1 & 5 & 1 & 1 & 1 & 1 \\
\hline Limpieza zona de descanso & 1 & 5 & 3 & 3 & 3 & 3 & 5 \\
\hline Condición corporal & 3 & 3 & 3 & 3 & 3 & 3 & 3 \\
\hline Infecciones locales & 5 & 5 & 5 & 5 & 3 & 3 & 5 \\
\hline Heridas en el cuerpo & 5 & 5 & 5 & 5 & 5 & 5 & 1 \\
\hline Cojeras & 5 & 5 & 5 & 5 & 5 & 5 & 5 \\
\hline Estiércol en el cuerpo & 5 & 5 & 3 & 3 & 5 & 5 & 3 \\
\hline
\end{tabular}

Criterios con valoración de 1 se determina como inadecuado (factor de riesgo); 3, neutral y 5, adecuado.

Morales [27] confirma que al no brindar un adecuado mantenimiento de desinfección a los bebederos, se aumenta la exposición de contagio de microorganismos patógenos y mortalidad de las aves. En su estudio, Rueda ${ }^{[28]}$ encontró que las aves que se abastecen de agua que contiene material particulado tienden a presentar síntomas de malestar y reducción de producción de huevo. Con estas observaciones se coincide con las reflexiones de Matute y Rivas [29], quienes señalan que el agua contaminada o sucia influye en el desarrollo del ciclo biológico de los parásitos y también se pueden convertir en vehículo de transmisión.

En la limpieza de las zonas de descanso se observó que los nidales de las gallinas se encontraban sucios y con abundantes heces, lo que puede favorecer una infección parasitaria. Esta información no sigue lo recomendado por Guinebretière et al. [30], quienes consideran que la limpieza de los nidales ayuda a reducir el contagio de enfermedades infecciosas y además evita que se presenten huevos sucios y la contaminación de la cáscara. Castañeda-Benjumea ${ }^{[31]}$ reporta que la limpieza general de los corrales ayuda a reducir el riesgo que se desarrollen y propaguen enfermedades en las gallinas y que esta actividad debe ser continua, como parte de las buenas prácticas de manejo.

El factor heridas en el cuerpo solo se encontró en el asno, al presentar hemorragias en su zona orbital, así como falta de limpieza de la misma, lo que posiblemente puede desencadenar una transmisión de agentes infecciosos por el contacto de la herida abierta en la piel con suelo u otros animales. Reyes et al. ${ }^{[32]}$ mencionan que una forma de contagio de un patógeno entre individuos puede ser a través del contacto de heridas de la piel. Acha y Szyfreses [33] señalan que cuando un indi- 
viduo presenta una herida que se puede infectar por el contacto con elementos contaminados de algún agente infeccioso, ya que este puede introducirse en los tejidos blandos del cuerpo.

No se detectó huevos de parásitos en el excremento del asno, sin embargo, el criterio heridas en el cuerpo se incluyó en la matriz debido a que se determinó como un factor de riesgo.

En la Tabla 5 se observan las prácticas determinadas como factores de riesgo sobre los animales de producción del RU-UACJ, mediante la lista de verificación.

TABLA 5

Prácticas Determinadas como Factores de Riesgo sobre los Animales de Producción del RU-UACJ MEDIANTE LA LISTA DE VERIFICACIÓN

\begin{tabular}{|c|c|c|c|c|c|c|c|}
\hline \multirow{2}{*}{ FACTORES DE RIESGOS } & \multicolumn{7}{|c|}{ ANIMALES DE PRODUCCIÓN } \\
\hline & GaLlinas & EQUINOS & Porcinos & Bovinos & Ovinos & CAPRINOS & Asno \\
\hline Animales nuevos sin cuarentena & $x$ & $x$ & $x$ & $x$ & $x$ & $x$ & $x$ \\
\hline No desparasitación & $x$ & $\times$ & $x$ & $x$ & $x$ & $x$ & $x$ \\
\hline Ausencia de pediluvio & $x$ & $x$ & $x$ & $x$ & $x$ & $x$ & $x$ \\
\hline Pastoreo mixto & $x$ & $x$ & - & $\times$ & $x$ & $x$ & $x$ \\
\hline Accesibilidad con fauna silvestre & $x$ & $x$ & - & $x$ & $x$ & $x$ & $x$ \\
\hline Animales muertos & $x$ & - & - & - & - & - & - \\
\hline
\end{tabular}

Símbolos: $\times$, se presenta la práctica (determinada como factor de riesgo); -, no se presenta práctica.

A la entrada de cada corral se evidenció la ausencia del tapete sanitario (pediluvio), sin embargo, en caprinos y el asno no se reportó presencia parasitaria, pero este factor puede afectar a todos los animales de producción del RU-UACJ. Además, no existe una zona específica de desinfección, lo que probablemente puede influir en una transmisión parasitaria. Esto no se ajusta a lo que establece la OIE ${ }^{[3]}$ sobre la importancia del uso del tapete sanitario como una medida de bioseguridad dentro de un establecimiento para minimizar el riesgo de propagación de agentes infecciosos. Calderón ${ }^{[34]}$ recomienda la utilización de pediluvios en las puertas de ingreso de los corrales, así como en otras áreas dentro de una granja.

En el transcurso de 2020 no se desparasitó a los animales con la frecuencia regular debido a que se presentó la pandemia de SARS-CoV-2 y los estudiantes de veterinaria de la UACJ no asistieron al RU-UACJ a realizar prácticas de apoyo concernientes al plan sanitario de los animales. Guagala [35] resalta que una explotación pecuaria debe incorporar dentro de sus prácticas habituales un sistema de desparasitación, puesto que ayuda a minimizar riesgos en la salud de los animales. También la SAGARPA y SENASICA [36], dentro de sus lineamientos recomiendan establecer un programa de control para endo y ecto parásitos, en función de la granja y análisis de laboratorio, ya que favorece al control sanitario.
La presencia de animales nuevos sin cuarentena previa es un factor que puede afectar a todos los animales de producción del RU-UACJ. Se observó que a los animales nuevos se les permite el ingreso a los rebaños siempre y cuando no presenten ninguna manifestación de malestar. Esta práctica no sigue con las recomendaciones de la OIE [3], la cual enfatiza que tanto las enfermedades como los parásitos se deben evitar y controlar a través de buenas prácticas de manejo animal y de tener en cuenta la importancia de aplicar un proceso de cuarentena a los animales de nuevo ingreso que deben pasar por un proceso de adaptación, ya que esta medida contribuye a la bioseguridad animal y pública. Daniel et al. ${ }^{[37]}$ destacan la importancia de someter a cuarentena los animales de nuevo ingreso al rebaño para descartar contagios de enfermedades infecciosas y reducir pérdidas económicas y muertes de los semovientes.

En cuanto al manejo de los animales muertos, en el corral de las gallinas se encontraron cadáveres de varios días dentro del gallinero y encima del techo. La eliminación de animales muertos debe de atenderse de forma inmediata ${ }^{[3]}$. Asimismo, Gekara y Leite-Browning [38] señalan que los productores de animales deben mantener prácticas de bioseguridad adecuadas en el manejo de animales muertos, ya que esto ayuda a evitar la introducción de organismos causantes de enfermedades que pueden afectar a otros animales. 
La accesibilidad con fauna silvestre es constante en los rumiantes, équidos y gallinas del RU-UACJ debido a la particularidad de los corrales y zonas de pastoreo, los cuales están delimitados, pero no son completamente restrictivos. En los porcinos este factor no se determinó como riesgoso debido a su constante confinamiento. En el caso de las gallinas existe una rotura de su corral, lo cual permite la interacción con otros animales.

Durante el estudio se constató la presencia de conejos silvestres (Sylvilagus audubonii), correcaminos (Geococcyx californianus), coyote (Canis latrans), gorrión doméstico (Passer domesticus), chanate mayor (Quiscalus mexicanus), huilota común (Zenaida macroura) y paloma doméstica (Columba livia). En este escenario se puede suponer una posible transmisión parasitaria entre animales silvestres y de producción. Jakob-Hoff et al. ${ }^{[1]}$ señalan que la interacción entre animales de granja y silvestres puede presentar un contagio directo de enfermedades cuando en estos contactos no se tratan con medidas adecuadas, ya que algunos animales son huéspedes intermedios y otros suelen ser portadores directos de microorganismos infecciosos. La OIE [39] menciona que cuando hay granjas que colindan o coinciden en parte con ambientes donde habitan animales silvestres, siempre este escenario dará lugar a un mayor contacto de agentes patógenos, lo que favorece a la circulación y propagación de enfermedades.

El pastoreo mixto no afecta a los porcinos, ya que los animales constantemente permanecen confinados en sus instalaciones y no se les permite la salida al exterior de sus corrales, mientras que para las otras especies animales se permite el pastoreo mixto cuando hay disponibilidad de forraje.

Guzmán et al. [40] mencionan en sus consideraciones que en zonas donde se presenta el pastoreo conjunto de rumiantes, los animales jóvenes podrían representar un riesgo de contagio parasitario de Haemonchus y Trichostrongylus, dada la susceptibilidad de ambos huéspedes. Sin embargo, contradictoriamente, Marley et al. [41] demostraron en su estudio que el manejo del pastoreo mixto permite reducir los niveles de contaminación parasitaria de las pasturas y que a su vez posibilitaba el control de nematodos.

\section{Evaluación de impacto de los factores de riesgo}

En la Tabla 6, mediante la matriz de Leopold combinada con los CRI, se observan los resultados de la evaluación de impacto de cada factor de riesgo, con un valor mínimo de 2.8 y máximo de 30.7. Los factores con una puntuación de 16 a 39 requieren de la adecuación de prácticas correctivas específicas de control en forma inmediata. Estos factores pueden llegar a perturbar de manera significativa la salud de los animales de producción. Los factores con valores de 0 a 5 no requieren de medidas correctivas específicas de control y tampoco inmediatez, pero sí precisan que sean atendidos con medidas correctivas básicas.

TABLA 6

Evaluación de Impacto de los Factores de Riesgo en cada Grupo Animal por medio de la Matriz Combinada con Criterios Relevantes Integrados

\begin{tabular}{|c|c|c|c|c|c|c|c|c|c|}
\hline ACCIONES & \multicolumn{9}{|c|}{ FACTORES DE RIESGO } \\
\hline $\begin{array}{l}\text { IMPACTANTES } \\
\text { DENTRO DEL } \\
\text { RANCHO } \\
\text { ANIMALES } \\
\text { AFECTADOS }\end{array}$ & 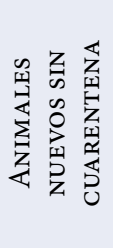 & z. & 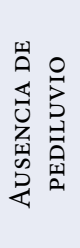 & 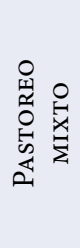 & 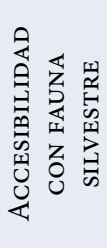 & 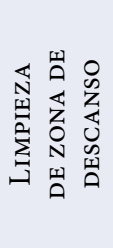 & 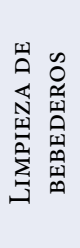 & 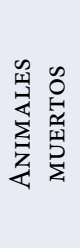 & 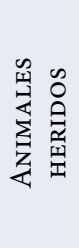 \\
\hline Gallinas & 30.7 & 29.1 & 29.1 & 26.6 & 24.4 & 21.5 & 16.0 & 16.0 & \\
\hline Equinos & 30.7 & 29.1 & 29.1 & 26.6 & 24.4 & & 4.0 & & \\
\hline Porcinos & 30.7 & 29.1 & 29.1 & & & & & & \\
\hline Bovinos & 30.7 & 29.1 & 29.1 & 26.6 & 24.4 & & 4.0 & & \\
\hline Ovinos & 30.7 & 29.1 & 29.1 & 26.6 & 24.4 & & 2.8 & & \\
\hline Caprinos & 30.7 & 29.1 & 29.1 & 26.6 & 24.4 & & 2.8 & & \\
\hline Asno & 30.7 & 29.1 & 29.1 & 26.6 & 24.4 & & 4.0 & & 16.0 \\
\hline
\end{tabular}

Escala de severidad: leve (1-5), moderado (6-15), severo (16-39), crítico (40-100). 
De la evaluación se obtuvo como resultado que ausencia de pediluvio, no desparasitación y animales nuevos sin cuarentena son factores que afectan a todos los animales de producción del RU-UACJ, representando un riesgo de $100 \%$ para cada uno (Figura 1), seguido de accesibilidad con fauna silvestre, pastoreo mixto y limpie$z a$ de bebederos con un $85.7 \%$ cada uno, sin afectar a los porcinos. Los factores animales muertos (14.3\%) perjudica a las gallinas, animales heridos (14.3\%) afecta solo al asno y limpieza de zona de descanso (14.3\%) perturba únicamente a las gallinas.

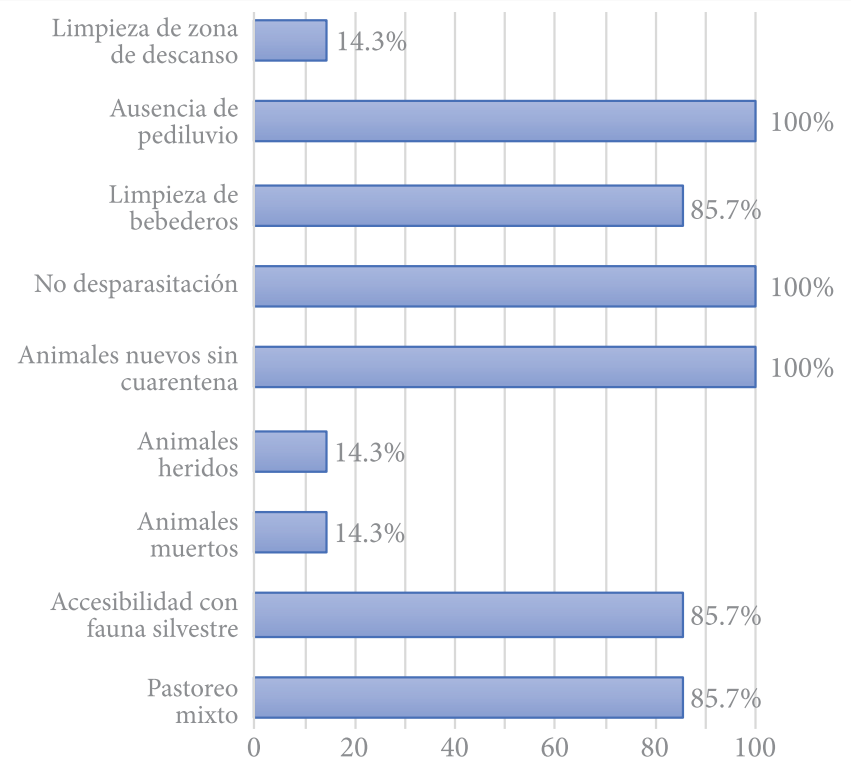

Figura 1. Porcentajes de los factores de riesgo sobre los animales de producción del RU-UACJ.

Los animales impactados con ocho factores de riesgos considerados como severos fueron las gallinas, con $88.8 \%$ (Figura 2); el asno, con 7 factores (77.7\%); los bovinos, ovinos, caprinos y equinos, con 6 factores (66.6\%), y los porcinos con 3 factores (33.3\%).

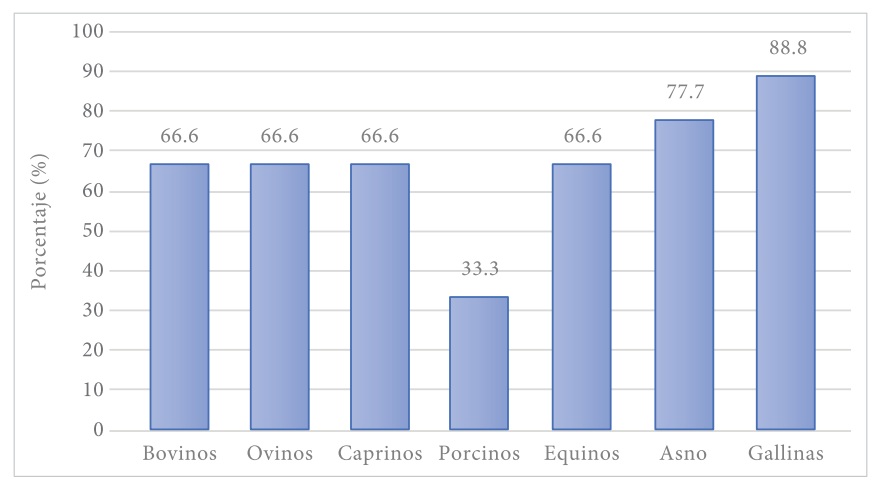

Figura 2. Porcentajes de riesgo por grupo animal.
En cuanto a los análisis de los factores de riesgo se concuerda con las siguientes entidades y leyes, quienes recomiendan y establecen medidas de control sanitario animal: La OIE [3] aconseja realizar análisis de riesgos con el propósito de establecer un control de puntos críticos en la sanidad animal. Asimismo, la Ley Federal de Sanidad Animal en México [42] establece medidas en materia de buenas prácticas pecuarias para reducir los riesgos zoosanitarios en establecimientos de tipo pecuario. Del mismo modo, la Norma Oficial Mexicana NOM-046-ZOO-1995 [43] especifica los criterios para la aplicación de la vigilancia epidemiológica de enfermedades que pongan en riesgo la salud animal. También la Secretaría de Agricultura y Desarrollo Rural (SAGARPA), junto con el Servicio Nacional de Sanidad, Inocuidad y Calidad Agroalimentaria (SENASICA) [36], ha diseñado manuales para fomentar la implementación de las buenas prácticas en producciones pecuarias con el fin de controlar las enfermedades que afectan a los animales.

\section{CONCLUSIONES}

Se identificaron seis géneros de parásitos con sus respectivas prevalencias en las muestras de excremento de los animales de producción del RU-UACJ: Trichostrongylus (66\%) y Strongyloides (16\%) en bovinos, Haemonchus (40\%) en ovinos, Strongyloides (66\%) en porcinos, Strongylus (100\%) y Cyathostomum (66\%) en equinos y Ascaris (100\%) en gallinas, en la temporada de verano de 2020. El grupo animal menos afectados fue el de los porcinos.

Los factores considerados como severos son limpieza de zona de descanso, que perturbó solo a las gallinas; la ausencia de pediluvio, la no desparasitación y contar con animales nuevos sin cuarentena, impactaron en todos los animales de producción del RU-UACJ; animales heridos, que afectó al asno; animales muertos, que perjudicó a gallinas; la accesibilidad a la fauna silvestre y el pastoreo mixto, que afectó a los rumiantes, équidos y gallinas. Estos factores de riesgos requieren de medidas correctivas específicas y de forma inmediata.

La matriz de Leopold, combinada con Criterios Relevantes Integrados, resultó útil para demostrar el impacto de los factores de riesgo en la salud de los animales de producción del RU-UACJ. 
E. G. Quiroga-Calderón et al. Factores de Riesgo Asociados a Parásitos Gastrointestinales en Animales de Producción ARTÍCULO DE INVESTIGACIÓN

\section{REFERENCIAS}

[1] R. M. Jakob-Hoff, S. C. MacDiarmid, C. Lees, P. S. Miller, D. Travis y R. Kock. Manual of procedures for wildlife disease risk analysis. (2014). [En línea]. Disponible en: portals.iucn.org/library/sites/library/ files/documents/2014-007.pdf

[2] J. H. Leibler et al., "Industrial Food Animal Production and Global Health Risks: Exploring the Ecosystems and Economics of Avian Influenza", Ecohealth, vol. 6, no. 1, pp. 58-70, mar. 2009. doi: 10.1007/s10393-009-0226-0.

[3] Organización Mundial de Sanidad Animal. "Código Sanitario para los Animales Terrestres (2019)". OIE. int. www.oie.int/es/que-hacemos/normas/codigos-ymanuales/acceso-en-linea-al-codigo-terrestre/ (consultado: enero 9, 2021).

[4] B. Wimmer, B. H. Craig, J. G. Pilkington y J. M. Pemberton, "Non-invasive assessment of parasitic nematode species diversity in wild Soay sheep using molecular markers", Int. J. Parasitol., vol. 34, no. 5, pp. 625-631, abr. 2004, doi: 10.1016/j.ijpara.2003.11.022.

[5] S. A. Squire, H. Amafu-Dey y J. Beyuo, "Epidemiology of gastrointestinal parasites of cattle from selected locations in Southern Ghana", Livest. Res. Rural. Dev., vol. 25 no. 7, jul. 2013, www.researchgate.net/ publication/287521798.

[6] K. VanderWaal, G. P. Omondi y V. Obanda, "Mixedhost aggregations and helminth parasite sharing in an East African wildlife-livestock system", Vet. Parasitol., vol. 205, nos. 1-2, pp. 224-232, sept. 2014, doi: 10.1016/j. vetpar.2014.07.015.

[7] S. Sahinduran, "Protozoan Diseases in Farm Ruminants", en A Bird's-Eye View of Veterinary Medicine, C. C. Perez-Marin, ed., IntechOpen, 2012, cap. 23, doi: $10.5772 / 30251$.

[8] A. Volpato et al., "Gastrointestinal protozoa in dairy calves: identification of risk factors for infection", Rev. MVZ Córdoba, vol. 22, no. 2, pp. 5910-5924, may.-ag. 2017, doi: 10.21897/rmvz.1027.

[9] V. Obanda, N. Maingi, G. Muchemi, C. J. Ng'ang’a, S. Angelone y E. A. Archie, "Infection dynamics of gastrointestinal helminths in sympatric non-human primates, livestock and wild ruminants in Kenya”, PLoS One, vol. 14, no. 6, jun. 2019, doi: 10.1371/journal.pone.0217929.

[10] S. Duffy, C. L. Burch y P. E. Turner, "Evolution of host specificity drives reproductive isolation among RNA viruses", Evolution, vol. 61, no. 11, pp. 2614-2622, nov. 2007, doi: 10.1111/j.1558-5646.2007.00226.x.

[11] INEGI. "Instituto Nacional de Estadística y Geografía”. INEGI.org. www.inegi.org.mx/ (consulta: junio 15, 2021).

[12] UACJ, Cuarto informe de actividades 2015-2016. Ciudad Juárez: UACJ. 2016. Disponible en: www3.uacj.mx/ comunicacion/Documents/Cuarto Informe completo. pdf.

[13] PANAFTOSA / OPS / OMS. Manual Veterinario de Toma y Envío de Muestras. (2017). [En línea]. Disponible en: iris.paho.org/bitstream/handle/10665.2/34527/ 01016970MT13spa.pdf? sequence=1\&isAllowed $=\mathrm{y}$

[14] OPS/OMS, Medios auxiliares para el diagnóstico de las parasitosis intestinales, 2. ${ }^{\mathrm{a}}$ ed. Organización Panamericana de la Salud, 2020. [En línea]. doi: 10.37774/9789275322055.

[15] S. Aguilar-Barojas, "Fórmulas para el cálculo de la muestra en investigaciones de salud", Salud en Tabasco, vol. 11 nos. 1-2, pp. 333-338, en.-ag. 2005, disponible en: www.redalyc.org/articulo.oa?id=48711206.

[16] OPS/OMS, Indicadores de salud. Aspectos conceptuales y operativos. Washington: Organización Panamericana de la Salud, 2018. [En línea]. doi: 10.37774/9789275320051.

[17] Welfare Quality, Assessment protocol for pigs. Lelystad, Países Bajos: Welfare Quality, 2009, pp. 1-123. [En línea]. Disponible en: www.welfarequalitynetwork.net/ media/1018/pig_protocol.pdf.

[18] C. Pérez, Uso de lista de cotejo como instrumento de observación, Serie Apoyo a la Docencia no. 1. Puerto de Manzanillo, México: Universidad Tecnológica Metropolitana, 2018, pp. 1-21. [En línea]. Disponible en: vrac.utem.cl/wp-content/uploads/2018/10/manua. Lista_Cotejo-1.pdf.

[19] Gayoso, J. Impacto ambiental de las prácticas de cosecha forestal y construcción de caminos en bosques nati- 
vos siempre verdes de la X Región de Chile, Serie Monografías de Explotación Forestal no. 6. Roma: FAO, 1995, 169 pp. [En línea]. Disponible en: www.fao.org/3/ v9727s/v9727s0a.htm\#7 identificacion y valoracion de impactos.

[20] J. G. Petters, "Prevalencias y cargas parasitarias en heces de Canis latrans, del APFF Médanos de Samalayuca", Tesis de maestría, Departamento de Ciencias Veterinarias, Universidad Autónoma de Ciudad Juárez, Ciudad Juárez, Chih., México, 2019.

[21] P. I. Maya, "Detección de huevos de helmintos en agua tratada, residual y de uso agrícola y evaluación de riesgo por género en Nuevo Casas Grandes, Chihuahua”, Tesis de maestría, Universidad Autónoma de Ciudad Juárez, Ciudad Juárez, Chih, México, 2017.

[22] A. Quezada, J. I. Ramo, C. Figueroa, R. R. Rivas, R. Martínez y V. Trillo, "Prevalencia de coccidia en becerras holstein en la etapa de desarrollo," Cult. Científ. y Tecnol., no. 49, esp. no. 2, en.-abr. 2013. [En línea]. Disponible en: erevistas.uacj.mx/ojs/index.php/culcyt/article/ view/186/179.

[23] J. P. Flores et al., Gastrointestinal diseases and causal effects in the Valle de Juárez, Chihuahua, México, col. Textos Universitarios, serie Investigación. Ciudad Juárez: Universidad Autónoma de Ciudad Juárez, 2010, 128 pp. [En línea]. Disponible en: bva.colech.edu.mx/xmlui/ handle/1/1701.

[24] O. González, "Prevalencia de parásitos en felinos silvestres en cautiverio", Tesis de licenciatura, Universidad Autónoma de Ciudad Juárez, Ciudad Juárez, Chih., México, 2012.

[25] M. Rivadeneira, "Diarrea en terneros por Coccidias", Monografía de grado, Universidad de Cuenca, Cuenca, Ecuador, 2012. [En línea]. Disponible en: dspace. ucuenca.edu.ec/bitstream/123456789/443/1/TESIS.pdf

[26] C. Aranda, E. Serrano-Martínez, M. Tantaleán, M. Quispe y G. Casas, "Identificación y frecuencia de parásitos gastrointestinales en félidos silvestres en cautiverio en el Perú", Rev. de Investig. Vet. del Peru, vol. 24, no. 3, pp. 360-368, ag. 2013, disponible en: www.scielo.org.pe/scielo.php?script=sci_arttext\&pid= S1609-91172013000300013.
[27] G. Morales, L. Arelis, E. Sandoval, D. Jiménez y J. Morales, "Relación entre la condición corporal y el nivel de infestación parasitaria en bovinos a pastoreo como criterio para el tratamiento antihelmíntico selectivo", Rev. Investig. Vet. del Perú, vol. 23, no. 1, nov. 2012, doi: 10.15381/rivep.v23i1.886.

[28] F. E. Rueda, "Rediseño de bebedero de agua para aves de corral con un sistema de filtro que reduzca el material particulado y sólidos en suspensión que contrae el agua en el ambiente externo de la zona rural del municipio de Palermo - Huila", Informe final de licenciatura, Universidad Cooperativa de Colombia, Neiva, Colombia, 2020. [En línea]. Disponible en: repository.ucc.edu.co/bitstream /20.500.12494/20140/1/2020_bebedero_agua_aves.pdf.

[29] M. L. Matute y W. I. Rivas, "Prevalencia de parásitos gastrointestinales según la época del año, en aves de patio jóvenes y adultas en el Sauce, León, Nicaragua”, Tesis de licenciatura, Departamento de Sanidad Animal / Unidad de Parasitología, Universidad Nacional Autónoma de Nicaragua, León, Nicaragua, 2012. [En línea]. Disponible en: riul.unanleon.edu.ni:8080/jspui/ bitstream/123456789/3311/1/225919.pdf.

[30] M. Guinebretière, A. Huneau-Salaün, D. Huonnic y V. Michel, "Cage hygiene, laying location, and egg quality: The effects of linings and litter provision in furnished cages for laying hens", Poult. Sci., vol. 91, no. 4, abr. 2012, doi: 10.3382/ps.2011-01881.

[31] C. Castañeda-Benjumea, "Evaluación del bienestar animal de gallinas ponedoras Bovans White alojadas en piso", Abanico Vet., vol. 10, no. 1, en. 2020, doi: 10.21929/ abavet2020.5.

[32] H. Reyes, P. Navarro y M. Sánchez, "Infecciones por parásitos en trabajadores de la salud: transmisión y control", Rev. Inst. Nac. Hig., vol. 35, no. 1, pp. 32-45, en. 2004, disponible en: ve.scielo.org/scielo.php?script=sci_ arttext\&pid=S0798-04772004000100006\&lng=es\&nrm $=\mathrm{iso} \& \mathrm{t} \operatorname{lng}=\mathrm{es}$.

[33] P. N. Acha y B. Szyfres, "Zoonosis y enfermedades transmisibles comunes al hombre y a los animales. Volumen I: Bacteriosis y micosis", Rev. Esp. Salud Pública, vol. 75, no. 3, pp. 263-264, en.-jun. 2001, doi: 10.1590/S113557272001000300009 . 
[34] K. S. Calderón, “Comparación del efecto desinfectante de hidróxido de calcio vrs. una mezcla de ácidos orgánicos y surfactantes, aplicados en pediluvios de una granja avícola en el departamento de Guatemala”, Tesis de licenciatura, Facultad de Medicina Veterinaria y Zootecnia, Universidad de San Carlos de Guatemala, Guatemala, 2005. [En línea]. Disponible en: www.repositorio. usac.edu.gt/id/eprint/7158.

[35] R. Guagala, "Prevalencia de parásitos gastrointestinales en bovinos en producción de leche del cantón Urcuquí", Tesis de licenciatura, Pontificia Universidad Católica del Ecuador, Ibarra, Ecuador, 2019. [En línea]. Disponible en: dspace.pucesi.edu.ec/handle/11010/420.

[36] SAGARPA/SENASICA. Manual de Buenas Prácticas Pecuarias en la Producción de Granjas Porcícolas, 2. ed. (2016). [En línea]. Disponible en: acsaa.com.mx/ wp-content/uploads/2019/06/Manual_de_Buenas_Pr_ cticas.pdf

[37] S. Daniel., M. A. Abeledo, I. Miranda y E. Lobo, "Identification of the risk factors for mortality by contagious bovine pleuropneumonia in Namibe, Angola”, Rev Salud Anim., vol. 38, no. 1, pp.1-8, 2016. [En línea]. Disponible en: scielo.sld.cu/scielo.php?script=sci_ arttext\&pid=S0253-570X2016000100001\&lng=es.

[38] O. Gekara, M. L. Leite-Browning, "Prácticas de Bioseguridad para los Ranchos y Granjas de los Pequeños Productores de Ganado Vacuno de Carne", Serie para productores de pequeña escala y aficionados [En línea], 2012. Disponible en: iiad.tamu.edu/wp-content/ uploads/2012/06/Small-Beef-Cattle-Part-1-Spanish.pdf

[39] OIE (Organización Mundial de Sanidad Animal), "Enfermedades de los animales silvestres", Hoja informativa [En línea], 2015. Disponible en: www.oie.int/fileadmin/ Home/esp/Media_Center/docs/pdf/Fact_sheets/WD_ ES.pdf

[40] M. Guzmán, C. Fiel, y P. Steffan, "La infección cruzada de Haemonchus contortus de ovinos a bovinos y el riesgo de transmisión de resistencia antihelmíntica.
Una revisión", Vet. Arg., vol. 27, no. 272, pp. 1-7, 2010, disponible en: www.produccion-animal.com.ar/sanidad_ intoxicaciones_metabolicos/parasitarias/parasitarias_ bovinos/141-Haemonchus_Contortus.pdf.

[41] C. L. Marley, M. D. Fraser, D. A. Davies, M. E. Rees, J. E. Vale y A. B. Forbes, "The effect of mixed or sequential grazing of cattle and sheep on the faecal egg counts and growth rates of weaned lambs when treated with anthelmintics”, Vet. Parasitol., vol. 142, no. 1-2, pp. 134-141, nov. 2006, doi: 10.1016/j.vetpar.2006.06.030.

[42] Cámara de Diputados. Sesión (2017, diciembre 12). Última reforma DOF 16-02-2018, Ley Federal de Sanidad Animal. [En línea]. Disponible en: www.diputados.gob. mx/LeyesBiblio/pdf/LFSA_160218.pdf

[43] SAGARPA. DOF. (1997, febrero 19). Norma Oficial Mexicana NOM-046-ZOO-1995, Sistema Nacional de Vigilancia Epizootiológica. [En línea]. Disponible en: www.gob.mx/cms/uploads/attachment/file/203473/ NOM-046-ZOO-1995_190297.pdf

\section{RECONOCIMIENTOS}

A la Universidad Autónoma de Ciudad Juárez y al programa de Maestría en Ciencia Animal por el desarrollo del trabajo de investigación.

Al Consejo Nacional de Ciencia y Tecnología (CONACYT) por la beca 748558 otorgada a Elkin Giovanny Quiroga Calderón durante el tiempo de estudios de la maestría (agosto de 2019 a julio de 2021).

Al Rancho Universitario UACJ y sus trabajadores por el apoyo a la realización del presente estudio.

Al Laboratorio de Ciencias Básicas de la División Multidisciplinaria de la UACJ en Nuevo Casas Grandes, donde se realizaron las técnicas coproparasitoscópicas.

A los compañeros de estudio Juan Rolando Rueda Torres, July Natalia Guerra Murcia y Bianca Viviana Orozco Galindo por el apoyo prestado en labores de campo. 